
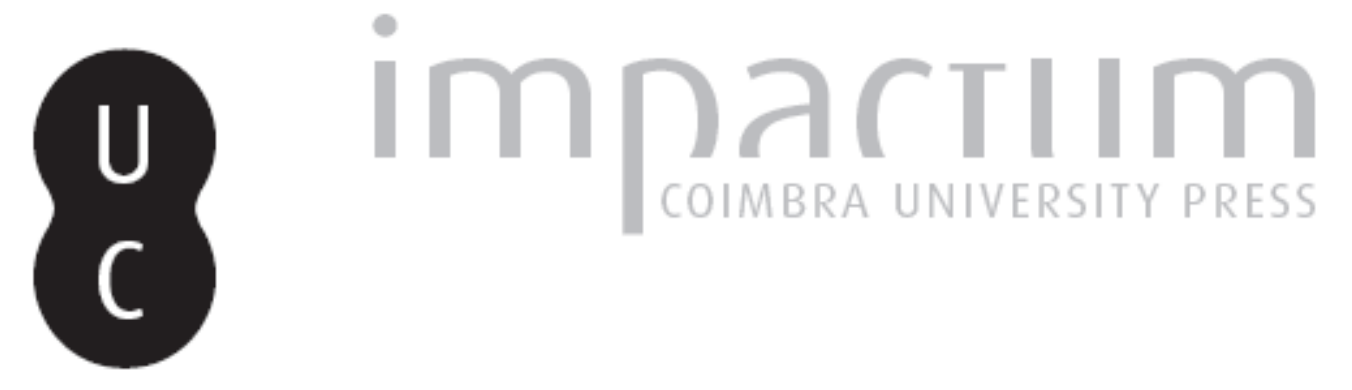

La prima rivoluzione in diretta

Autor(es): $\quad$ Castellina, Luciana

Publicado por: Imprensa da Universidade de Coimbra

URL persistente:

URI:http://hdl.handle.net/10316.2/42407

DOI:

DOl:https://doi.org/10.14195/0870-8584_9_1

Accessed : $\quad$ 26-Apr-2023 12:55:56

A navegação consulta e descarregamento dos títulos inseridos nas Bibliotecas Digitais UC Digitalis, UC Pombalina e UC Impactum, pressupõem a aceitação plena e sem reservas dos Termos e Condições de Uso destas Bibliotecas Digitais, disponíveis em https://digitalis.uc.pt/pt-pt/termos.

Conforme exposto nos referidos Termos e Condições de Uso, o descarregamento de títulos de acesso restrito requer uma licença válida de autorização devendo o utilizador aceder ao(s) documento(s) a partir de um endereço de IP da instituição detentora da supramencionada licença.

Ao utilizador é apenas permitido o descarregamento para uso pessoal, pelo que o emprego do(s) título(s) descarregado(s) para outro fim, designadamente comercial, carece de autorização do respetivo autor ou editor da obra.

Na medida em que todas as obras da UC Digitalis se encontram protegidas pelo Código do Direito de Autor e Direitos Conexos e demais legislação aplicável, toda a cópia, parcial ou total, deste documento, nos casos em que é legalmente admitida, deverá conter ou fazer-se acompanhar por este aviso. 


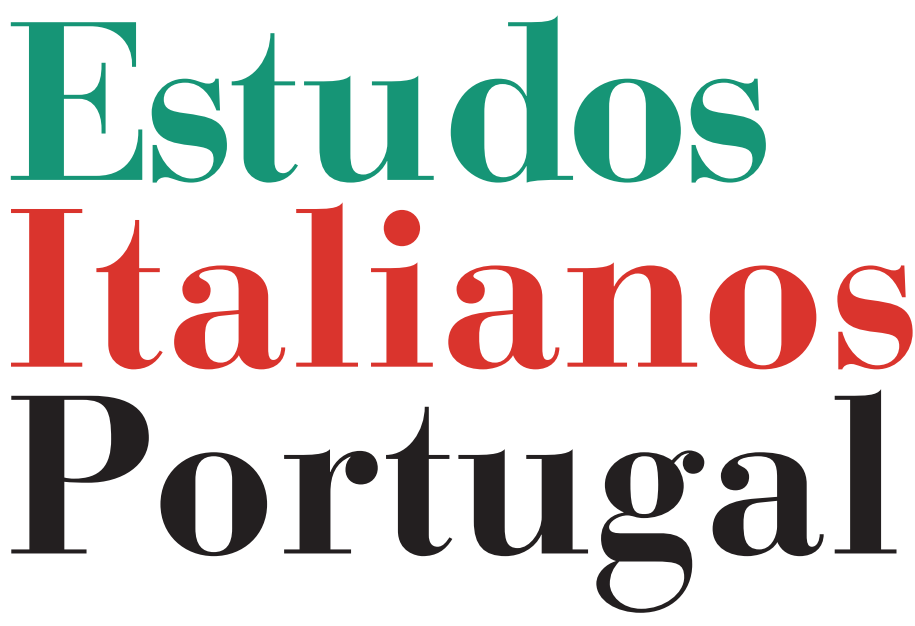

Instituto

Italiano

de Cultura

de Lisboa

Nova Série

$\mathbf{N}^{\circ} 9$ 


\section{LA PRIMA RIVOLUZIONE IN DIRETTA}

Luciana Castellina*

Oggi le avrebbero chiamate "primavere" - primavere sud europee. Come per quelle arabe anche allora si trattò di una sequela, una innescata sull'altra: la caduta del fascismo in Portogallo anticipò infatti di poco quella in Grecia, e poi, dopo un lasso di tempo appena più lungo, quella in Spagna. Un processo di democratizzazione che pose fine alla pagina oscura della storia del nostro continente che anche dopo la seconda guerra mondiale e la vittoria sul nazismo aveva tollerato due dittature, e poi aveva consentito che un'altra fosse rianimata, quella dei colonnelli greci nel ' 67.

E però fu il Portogallo ad iniziare, e nel modo più inaspettato e senza precedenti. Quando i garofani furono issati sulle canne dei fucili e imparammo Grandola vila morena, e accadde - il 25 aprile di 40 anni fa - ciò che accadde, facemmo persino fatica a capire, tanta fu la sorpresa.

"Sorpresa divina", la definirono gli stessi portoghesi che di quella rivoluzione anomala furono $i$ beneficiari ma che ai più giungeva del tutto inaspettata. Di rivoluzioni, o resistenze

* Luciana Castellina, giornalista, scrittrice e militante politica è stata esponente di diverse tendenze della sinistra italiana. Ha assunto vari incarichi politici ed è stata Parlamentare europea dal 1984 al 1999. Cofondatrice de "Il Manifesto", fra le sue ultime pubblicazioni si segnalano La scoperta del mondo (Nottetempo, 2011), finalista del premio Strega, resoconto della sua formazione comunista e Siberiana (Nottetempo, 2012), cronaca di un viaggio in treno da Mosca a Vladivostok sulla Transiberiana. 
popolari, il paese, del resto, non ne aveva fatte mai, diverso dalla Grecia e dalla Spagna dove guerre civili e di liberazione dall'occupazione nazista erano esperienze vissute. Non c'era mai stato, insomma, nessun apprendistato di massa, l'antifascismo era stato clandestino, fatto di prigioni, torture, esili, non di insurrezioni, brigate partigiane, manifestazioni di strada.

E' lontano da Lisbona, infatti, che questa rivoluzione era stata covata, in Africa. E da soggetti che di solito fanno colpi di stato, i militari, ma che poi, il potere conquistato anche con le migliori intenzioni, non sono abituati a cederlo al popolo, come invece capitò qui.

$\mathrm{Ci}$ andai subito a Lisbona, a vedere la meraviglia: pochi giorni dopo. Ma io per il mio giornale - Il Manifesto - mi occupavo da sempre della Grecia, dove al momento del colpo di stato ero stata persino arrestata e poi espulsa, e in quei giorni anche lì stava cominciando a ribollire la situazione che avrebbe portato alla caduta dei colonnelli. Perciò in Portogallo andai per un paio di giorni per mia curiosità, ma fu Rossana Rossanda che seguì per il giornale la prima fase della rivoluzione. Ricordo ancora quando tornò e ci raccontò della terra lusitana liberata dove era andata non solo per un reportage ma perché invitata proprio da quegli ufficiali della rivoluzione dei garofani a tenere una conferenza nel loro circolo: ci riferì, stupefatta, e divertita, del capitano Melo Antunes e del maggiore Tomè, dei graduati del settimo cavalleria, in divisa e stivali, che l'interrogavano su Gramsci: colti, educati, come di militari non ne avevamo visti mai e che però erano cresciuti nelle caserme degli ultimi, miserandi bastioni dell'impero in disfacimento, dove era giunto, attraverso i più giovani ufficiali di leva che avevano studiato nelle università europee, l'eco del '68.

A me toccò la seconda fase della rivoluzione - quella che seguì il tentato golpe del generale Spínola, l'11 marzo '75, che aveva creduto possibile dare al problema coloniale uno sbocco alla "francese", un'uscita con onore dell'esercito, che 
però garantisse alla metropoli i suoi interessi imperiali. Non aveva capito che i "capitani" che erano insorti erano sì motivati dalla guerra africana senza speranze, e terzomondisti nell'anima, ma che proprio per questo volevano cambiare anche il Portogallo, "il più avanzato dei paesi sottosviluppati" - ricordo che dicevano, ipotizzando un rapporto speciale con le ex colonie, in nome di una prospettiva per nulla "occidentale".

Spínola fu travolto dopo appena cinque mesi dal radicalizzarsi della situazione, e a settembre il Consiglio della Rivoluzione decretò, nel salone dell'Accademia di sociologia militare, che la depositaria della legittimità rivoluzionaria sarebbe stata l'assemblea dell'MFA, il Movimento delle Forze Armate: 60 delegati dell'esercito e altrettanti a ciascuna delle altre armi, marina e aviazione. Il loro primo atto fu la nazionalizzazione delle banche e delle assicurazioni.

A Lisbona, assieme a centinaia di giornalisti, operatori televisivi, fotoreporter provenienti da tutto il mondo ("La prima rivoluzione in diretta" - fu chiamata quella portoghese) rimasi molti mesi, fino alla fine della parabola rivoluzionaria, il 25 novembre. Un periodo straordinario, un susseguirsi di emozioni - entusiasmi e disillusioni - grazie ad una partecipazione e ad una inventiva popolare mai viste; e che infatti attrassero anche i giovani di tutta Europa.

Lisbona diventò la capitale dei sessantottini, ognuno dei quali si sentì portoghese, portando nell'agorà di quel tempo concitato le proprie ingenuità; e i propri incontenibili entusiasmi.

Anche io sono "diventata portoghese"; e per molti versi lo sono rimasta, anche perché questo paese ho continuato ad amarlo, per via delle amicizie strettissime stabilite allora e della mia funzione istituzionale: deputato al parlamento Europeo negli anni '80 feci parte della commissione incaricata di seguire la preparazione dell'ingresso nella Comunità Europea del Portogallo. 
Della rivoluzione dei garofani conservo due reperti: uno, tuttora attaccato alla parete della mia stanza, è la copertina de $O$ Jornal; l'altro, un biglietto sdrucito, vergato a matita da Otelo de Carvalho, con cui, dalla caserma nella quale era stato appena posto agli arresti, mi avvertiva che il nostro appuntamento era rinviato. Fra l'una e l'altro sono racchiusi i due estremi dell'epopea portoghese, un anno e mezzo anomalo, anzi bizzarro, dall'aprile ' 74 all'autunno '75. Poi tutto rientrò nella normalità.

La copertina di O Jornal raffigura i leader rivoluzionari di tutti i tempi seduti sui banchi di scuola: da Lenin a Mao a Gramsci a Bakunin a Trotzki, senza dimenticare né Stalin né Rosa Luxemburg, ma anche Marcuse e Sartre e Bertrand Russell, il volto assorto di chi è alle prese con un rompicapo. Alla lavagna la carta del Portogallo sovrastata da un punto interrogativo: che diavolo è questa rivoluzione condotta da militari che hanno letto Marx, non uno dei soliti colpi di stato militari, magari progressisti e però autoritari, come tanti altri nel terzo mondo, ma un'apertura di credito piena e persino eccessiva ad ogni possibile esperienza sociale di base, una straordinaria multiforme politicizzazione non solo consentita ma anche sollecitata, uno slogan, "o poder para o povo", che è anche un progetto - il "piano-guida" - di democrazia diretta? Per tutti, un rebus.

Il vero enigma, la specificità della vicenda, era in effetti questo MFA, Movimento delle Forze Armate, che, allontanato il reazionario colonnello Spínola, aveva preso le redini del paese avviando una serie di processi inediti nella storia dei socialismi realizzati. Ed è ben comprensibile che l'esperienza che si metteva in moto avesse affascinato la nuova sinistra di tutta Europa, in quegli anni alla ricerca di una strada che non ripercorresse gli errori sovietici ma nemmeno segnasse la rinuncia a combattere il capitalismo come aveva fatto la socialdemocrazia.

Qualcuno scrisse, con malignità, che andavano a consolarsi delle sconfitte subite nei rispettivi paesi dopo l'onda del 
"maggio". E, aggiunsero, che si trattava "della prima rivoluzione turistica”. E' ingeneroso: andarono, andammo, perché la fine del regime di Caetano e dell'ultimo presidio coloniale era grande festa e indicava che ovunque il mondo stava cambiando. Ed era in gran parte vero. Del resto chi andò si assoggettò a un regime di totale milizia: assemblee fino a notte tarda, cortei, documenti, nessuno quell'estate riuscì a farsi un bagno nelle spiagge adiacenti. Andarono a Lisbona non per turismo politico, ma perché valeva la pena seguire da vicino, capire. E i primi ad esserne contenti furono proprio i portoghesi che rompevano dopo mezzo secolo il loro isolamento ed erano affamati di dialogo.

All'aeroporto, accanto ai tabelloni con gli orari di arrivo e di partenza degli aerei, per mesi e mesi ci furono, fissi, gli avvisi con le convocazioni del giorno dei vari raggruppamenti politici europei, affinché chi arrivava potesse subito orientarsi (in tutte le lingue, meno che in spagnolo: Franco era ancora al potere e i ragazzi che pure accorsero a migliaia dal paese vicino erano obbligati a nascondere il volto nelle manifestazioni e a non dare appuntamenti nazionali).

Gli italiani erano come sempre esorbitanti: ricordo ancora le scritte: "Lotta continua si riunisce alle 22", "il PDUP-Manifesto alle $21 \ldots .$. , e così via. Tutti al camping sulla collina, fra gli alberi del grande parco di Monsanto, "vantaggio collaterale" del salazarismo che, combattendo il progresso, aveva risparmiato Lisbona dalla moderna speculazione edilizia. In quel bosco, poco graditi vicini, condividevano lo spazio con i primi contingenti di pieds noirs poveri e rancorosi, rimpatriati dalle colonie liberate in gran fretta dopo il 25 aprile.

Si discuteva fino all'alba e ci si incontrava con i nuovi compagni che la rivoluzione dei garofani ci aveva inaspettatamente offerto. Noi de Il Manifesto vedevamo quelli del MES, il movimento della sinistra socialista, per i quali il nostro comunismo eretico costituiva un punto di riferimento. Fra loro Cravinho, poi ministro dell'economia e Jorge Sampaio 
(che venne anche a Bologna al nostro congresso nazionale del 1975), in seguito, dal 1996 al 2006, nientemeno che presidente della Repubblica Portoghese.

I comunisti ufficiali si vedevano in realtà assai poco, sebbene per una fase abbiano pesantemente contato (e non per il meglio). Questa storia della democrazia diretta e dal basso non era pane per i loro denti. Per altre ragioni, neppure per quelli dei socialisti, che oltretutto venivano sospettati di essere la longa manu dell'ambasciatore americano Carlucci (il dramma del Cile si era appena consumato, lasciando sul terreno un clima appestato di sospetti).

Solo l'MFA accettava i mille piccoli soviet che erano nati ovunque: nei quartieri (i potenti comitati dei moradores), nell'esercito (i SUV, "soldati uniti vinceremo"), l'autogestione delle fabbriche, degli alberghi, dei giornali, dove i tipografi mettevano in discussione la divisione del lavoro e chiedevano di aver voce in capitolo, come i giornalisti, sulla linea della pubblicazione (questa fu la vicenda dell'occupazione del quotidiano República, non l'effetto di un ordine venuto da Cunhal).

Lisbona era una città meravigliosamente insubordinata, provocatoria, i marmorei, bianchi monumenti salazariani dissacrati dalle scritte rosse del grande giornale murale, animato da centinaia di mani, in cui la capitale si era trasformata: falci e martello e umorismo antiautoritario ("abbasso la cupola viva la copulazione", leggo in una vecchia foto scattata allora; e, sfottente, sovrascritta sui manifesti dei moderati che minacciosamente annunciavano che loro erano milioni, e sarebbero cresciuti: "prendete la pillola". E poi le code per vedere i film proibiti che venivano sequestrati ancora persino in Italia, trent'anni di dopoguerra ingoiati tutti in una volta, più il ' 68 , che per un momento sembrò realizzato, la fantasia al potere, i marinai con il fazzoletto rosso al collo, i soldati che ubbidivano ai loro delegati anziché ai graduati e venivano chiamati a risolvere tutto, persino le liti familiari. Tanto 
che il I reggimento artiglieria leggera addetto all'ordine della città, il celebre Ralis, era stato ribattezzato " 115 ”, il numero che corrispondeva al pronto intervento.

E poi i cortei, quasi quotidiani, praticamente il commento della città ai fatti del giorno: un voto all'assemblea dell'MFA, un passaggio o un rimpasto di governo (furono continui), una controversia alla Costituente: ogni evento era sollecitazione a scendere in strada, a far prendere la parola a quella nebulosa chiamata movimento e che accanto ai portoghesi vedeva, protagonisti come se il Portogallo fosse di tutti, italiani francesi inglesi tedeschi e così via (gli spagnoli, come ho detto, numerosissimi, ma col passamontagna). I giornali, bruttissimi perché stracolmi di slogan, a ruba: persino sulla spiaggia, quando durante il weekend i lisbonesi vi si riversavano perché la domenica non si fa la rivoluzione, tutti col naso dentro i fogli spiegazzati dal vento forte dell'Atlantico.

Non dipendeva solo dal '68, ma anche dalla speciale tradizione del movimento operaio portoghese, che aveva letto più Bakunin che Marx, e dell'Italia conosceva Malatesta meglio di Gramsci o Turati. Lo stesso Partito comunista qui, nel '21, non era nato - come ovunque altrove - dal Partito socialista, la cui influenza non era mai andata oltre qualche avvocato di provincia, ma dalla anarchica "Organizzazione operaia nazionale". Ed era naturale che questa cultura invadesse anche l'esercito rivoluzionario: i soldati tutti capelloni, uniformi improbabili, una banda di hippies, quasi a voler recuperare una stagione che il fascismo aveva negato alla loro generazione. Quando alcuni reparti furono destinati all'Angola, dove il passaggio all'indipendenza non era stato ancora assicurato, furono gli stessi militari in divisa a distribuire i volantini che incitavano alla diserzione.

Come sono poi andate a finire le cose è storia nota. Anche fra di noi - giornalisti e militanti di sinistra (le due cose spesso si sovrapponevano) - discutemmo nei mesi di declino della rivoluzione su come giudicare il rapidissimo succedersi degli eventi. Ricordo le lunghe telefonate fra Lisbona e 
la redazione a Roma: come valutare il documento varato il 25 marzo dai nove ufficiali "ragionevoli", autore principale Melo Antunes, il più saggio degli ufficiali (ma firmato anche dall'assai radicale Otelo), con cui si prendevano le distanze dalla fatale deriva "putchista" che il generale Gonçalves, l'austero primo ministro legato all'ala militare del Partito comunista, sembrava aver innescato nel tentativo di rispondere agli attacchi sempre più scoperti che il resto dell'Europa (incluso il prudentissimo PCI del compromesso storico) muoveva all'esperienza portoghese? Come isolare il gauchismo estremo dei tanti gruppi maoisti e marxisti-leninisti, che aveva persino espulso il partito comunista perché aveva difeso l'assemblea costituente e si era azzardato, nel momento più preoccupante della situazione, a cercare la collaborazione con i socialisti, del cui leader loro gridavano nei cortei "A morte Soares pagliaccio"? Bisognava rassegnarsi a seppellire l'anomalia dei garofani, c'erano altre strade percorribili ?

Ma i pericoli non andavano cercati solo nelle trame che i servizi segreti delle capitali potenti o il quartier generale della Nato certo allestivano. Emersero via via dai torbidi sussulti di una società che non era tutta rappresentata dalla libertaria Lisbona o dall'Alentejo rosso dei braccianti che avevano conquistato la terra, ma anche dal nord del "minifundo", della piccola proprietà contadina, dalla conservatrice Porto, abitata da una borghesia legata a filo doppio, anche per via del commercio dei vini, con l'Inghilterra. Inizialmente rinviata per effetto delle risorse offerte dal "tesoro" del vecchio dittatore, che in un paese poverissimo aveva nascosto sotto il materasso 850 tonnellate di lingotti d'oro, la crisi economica, aggravata dal boicottaggio e dalla fuga di capitali, cominciava a mordere soprattutto qui. Nella società profonda non erano state conquistate casematte e i militari più radicali ne avevano sottovalutato l'importanza. Si cominciava a capire che il povo non è necessariamente rivoluzionario, può anche essere sanfedista.

Il primo, drammatico segnale d'allarme arrivò a ferragosto. 
Fu a tarda sera che noi giornalisti fummo avvertiti che ad Alcobaça, $100 \mathrm{~km}$ a nord di Lisbona, c'erano scontri a fuoco. Accorremmo in tanti. Quando arrivammo dovemmo a fatica farci strada fra reparti dell'esercito provenienti dalle caserme di Caldas da Rainha e di Leira, politicamente rimaste lontane dall'MFA, e una folla di contadini armati di forconi e anche di armi da fuoco, aizzati da squadraccie di provocatori prezzolati. Circondavano il grande centro sportivo, isolato sulla cima della collina, dove si era riunito, per una sessione straordinaria, il Comitato centrale del PCP. Volevano assaltare l'edificio e cacciare quelli che ritenevano volessero strappargli la terra. Asserragliati nella palestra i comunisti, anche loro armati, rimasero per ore incerti se rispondere e aprirsi un varco, o accettare la mortificante difesa del conservatore comandante Charais, chiamato a salvarli da un popolo minaccioso.

La tensione si protrasse per ore, interrotta più volte da fucilate sparate dall'una e l'altra parte, noi giornalisti sotto le auto per ripararci dalle pallottole vaganti. Poi, in un'alba livida, la resa del PCP, inevitabile. In più di duecento furono caricati sulle camionette dell'esercito e portati in salvo, fra due ali di contadini trattenuti a stento dal linciaggio e di ironici militari. Un'immagine drammatica che per sempre mi rimarrà impressa: il volto scuro del vecchio Álvaro Cunhal, l'irriducibile stalinista dagli occhi azzurri e i capelli argentei, che proprio nella prigione di Peniche, a poca distanza, era stato rinchiuso per anni e anni, sette in isolamento, e anche appeso per i piedi, per giorni, nella cella, sempre in carcere mai in esilio, come è sempre stata la sorte dei comunisti.

Gli assalti alle sedi del PCP nel nord si susseguirono, sotto gli occhi di una parte delle forze armate che né attaccavano né difendevano, si limitavano a umiliare i militanti, come nella tradizione portoghese si fa coi tori, che non si ammazzano. Perché nel frattempo si era spezzata l'unità dell'MFA, le cui diverse correnti si combatterono per mesi, sia pure senza mai 
usare la forza di cui pure tutti disponevano, mentre cresceva l'isolamento del governo Gonçalves, sempre più ossessivamente impegnato a infiltrare $\mathrm{i}$ suoi fidi in tutti i possibili gangli del potere, mentre precipitosamente crollava la sua egemonia nella società.

Persino il PCP cominciò a rendersi conto che era da irresponsabili procedere allo scioglimento dell'Assemblea Costituente come il generale primo ministro a un certo punto propose. Ma era tardi, i modi antidemocratici con cui Cunhal gestiva il sindacato avevano logorato la loro credibilità nelle fabbriche, gli operai si disinteressarono di quanto accadeva, mentre impazzivano nell'estremismo le sei organizzazioni che avevano dato vita al Fronte Unito Rivoluzionario, da cui avevano espulso il PCP, giudicato "capitolardo", perché si era finalmente deciso a proporre un rapporto con i socialisti. Sornione, Mario Soares rimaneva in disparte, aspettando momenti migliori per far valere quel $38 \%$ di voti guadagnato alle elezioni che poco o niente contavano nel determinare il potere reale. Ricordo in quelle drammatiche settimane il titolo di un giornale: "Il PS è forse andato in ferie?".

Il 21 agosto uno degli ultimi grandi sussulti positivi: contro i rischi di una spaccatura dell'MFA, e soprattutto fra povo e Forze armate, un grande corteo, più di 50.000 da São Bento a Belém, la sfilata aperta da mille soldati e marinai che salutano la folla col pugno chiuso, gli operai delle fabbriche siderurgiche di Setúbal in tuta e elmetto di lavoro, i proletari della periferia a bordo dei camion che l'esercito aveva fornito, i contadini delle nuove cooperative del sud sui trattori, una foresta di bandiere rosse. Alla testa un lungo striscione su cui erano dipinti due sinistri signori: l'imperialismo americano e quello sovietico. La scritta: "contro l'imperialismo, unità col terzo mondo".

Nel corteo nessun vessillo di partito, ma tutta la sinistra presente, anche i comunisti ultraortodossi del PCP nonostante l'attacco all'URSS, e nonostante gli slogan dei gruppi 
gauchisti contro "la banda cunhalista che appoggia i governi borghesi”.

Il PCP subisce perché, più avvisato degli altri, è preoccupato della ormai evidente spaccatura nei vertici dell'MFA. L'Assemblea del movimento non riesce infatti a riunirsi per votare e tutti attaccano tutti: Otelo manda una lettera al generale Gonçalves, ancora presidente di un moribondo governo, piena di citazioni di Mao Tse Tung sul rapporto con le masse e gli proibisce di mettere piede nei reparti sotto il suo controllo, suggerendogli di andare in ferie per curarsi i nervi. Il presidente Costa Gomes, che tenta la mediazione, inventandosi una formula dietro l'altra, viene accusato di essere "la sinistra della Nato", o "la destra della rivoluzione". Il vecchio generale, il solo ad avere questo grado prima del 25 aprile, si rende conto che deve mettere assieme un governo meno impopolare e giacobino di quello diretto da Gonçalves, andando incontro alle posizioni prudenti di Melo Antunes, ma teme che nessuno sia poi in grado di controllare le fazioni militari che potrebbero finire per usare la forza l'una contro l'altra. E così finisce per nominare proprio Gonçalves, dopo aver affidato la formazione di un nuovo governo all'ammiraglio Pinheiro de Azevedo, capo di stato maggiore dell'esercito. Quasi una provocazione. Persino il PCP, cui pure Gonçalves è legato, si stupisce della decisione.

Il 12 settembre, mentre in città si moltiplicavano i boatos su possibili colpi di mano dell'una o dell'altra fazione, l'appuntamento decisivo per la storia era a Tancos, a $128 \mathrm{~km}$ da Lisbona, alla scuola pratica di ingegneria del genio. Al cancello cui approdammo noi giornalisti - ricordo - un ultimo segno della stagione dei garofani: un allievo ufficiale presta servizio, mano nella mano con la fidanzata che l'è venuto a trovare. $\mathrm{Ci}$ smista gentile in una sala per farci seguire i lavori dell'assemblea dell'MFA, il parlamentino del movimento che dovrà trovare una soluzione e colmare il vuoto di potere che si è creato. Ma quello cui assistiamo è il funerale dell'anomalo 
movimento delle forze armate: partecipano solo i gonçalvisti e pochi altri, una minoranza, nonostante la presenza di un riluttante presidente della Repubblica, il generale Costa Gomes. Gli altri, la destra, ma anche la maggioranza che si riconosce nel documento dei nove e che aveva visto mesi prima la convergenza fra l'irruento Otelo de Carvalho e il prudente Melo Antunes, si sparpaglia per i prati circostanti, o torna nelle proprie caserme.

Per settimane lo scontro fra le fazioni resta incruento, sembra una "battaglia navale", il gioco che si svolge sulla carta a quadretti. Poi però le cose precipitano perché l'insubordinazione dilaga. L'Assemblea dell'MFA muore così a sei mesi dalla sua costituzione, e con lei il progetto di potere popolare, varato il 9 luglio, il disegno di uno stato controllato da forme di democrazia diretta che non si era capito se dovesse affiancare o sostituire le istituzioni parlamentari. Proprio contro questa fuga in avanti, il 25 luglio, c'era stata la reazione dei nove, con il documento che aveva visto sancita, per una fase almeno, l'unità dei principali protagonisti della Rivoluzione, preoccupati dell'irrealismo delle frange estremiste delle forze armate.

In questo contesto la figura più contraddittoria, ma anche più popolare e generosa, quella di Otelo de Carvalho, capo dell'ala intransigente, il COPCON, è la più difficile da spiegare. Ho mancato - come ho detto - l'ultimo appuntamento che avevo con lui. Ma l'avevo incontrato spesso. Non era certo un uomo incerto, anzi irruento. E però alla fine tentenna: prima capisce che occorre guardarsi dall'estremismo, poi però prende il sopravvento il suo timore che lo stesso documento dei nove, da lui stesso firmato, sia usato in modo distorto per dar spazio alla destra. Un timore non infondato, e che però lo getta nelle braccia dei gruppi radicali più insensati. Che a partire da novembre innescano un'escalation, trovando comunque consenso fra una parte dei comitati, soprattutto operai, che arrivano a sequestrare il primo ministro 
dentro il palazzo del governo. Otelo, che pure non è responsabile delle iniziative più estreme, contro cui pure non vuole infierire, finisce per diventare il caprio espiatorio: destituito dal Consiglio della Rivoluzione da comandante della regione militare di Lisbona, la situazione gli sfugge di mano, sebbene non sia la guida, come pure venne accusato, della insorgenza delle truppe che si ribellano alla decisione e che con l'appoggio dei paracadutisti tentano un vero golpe. Invitato a prendere la direzione del pronunciamento dagli insorti, rifiuta e ubbidisce al presidente della Repubblica, Costa Gomes, che l'ha convocato (ma questo, come è noto, non gli ha impedito arresti, particolarmente pesanti quando, anni dopo, fu - credo - ingiustamente accusato di essere coinvolto in una dissennata avventura terrorista promossa da gruppi di estrema sinistra).

Ancora una volta i giornalisti hanno di che stupirsi sul Portogallo: dopo tre giorni di scontri i ribelli vengono sconfitti e arrestati, ma al prezzo di soli 3 morti, quando tutto lasciava pensare ad una carneficina. La vicenda del Portogallo non è dunque finita in tragedia, come avrebbe potuto. Per merito di tutti, in definitiva, ma innanzitutto dell'MFA che, nonostante le divisioni, riuscì a evitare lacerazioni cruente $\mathrm{e}$ a impedire si creasse lo spazio per pericolose avventure.

Negli anni ottanta ho continuato a seguire le vicende del paese, in tutt'altra collocazione: come membro della commissione mista Parlamento europeo - Parlamento portoghese che ha preparato l'ingresso di Lisbona nell'Unione Europea. Nonostante le vittorie elettorali della destra, a più riprese, il segno della stagione dei garofani si percepiva anche allora. Significativa la vittoria elettorale del PRD, il partito appoggiato dal presidente della Repubblica Eanes e ispirato da Melo Antunes, che raccolse tutta l'élite dell'MFA che rimase sempre estranea al potere politico, riuniti dopo tante divisioni e che ottenne il 18 per cento dei voti. Così come il consenso raccolto dalla candidata alla presidenza, nel 1986, 
Maria de Lurdes Pintasilgo, una singolare figura indipendente sostenuta dall'“Associazione 25 aprile", espressione diretta di quanto era sopravvissuto dell'MFA. Sono restata stupita nel leggere che nel 2012 Vasco Lourenço, uno dei principali protagonisti della rivoluzione dei garofani e uno dei firmatari del famoso documento dei nove assieme a Melo Antunes ma anche ad Otelo, ora presidente dell'Associazione, ha rifiutato di partecipare alle celebrazioni del 25 aprile in Parlamento per via della politica di austerità imposta dal governo di destra al Portogallo. Un gesto cui si è associato il vecchio presidente socialista Soares, che dell'MFA non fu certo mai un simpatizzante.

Del resto, sono stata contenta, il 12 ottobre del 2003, di sfilare assieme a lui, di cui confesso di aver pensato il peggio nel '74 e nel '75, nella marcia della pace, la Perugia-Assisi. Contro la guerra all'Irak senza si e senza ma. 